\section{Mapping metastases}

\section{By Joanne Kotz, Senior Editor}

Memorial Sloan-Kettering Cancer Center, Cornell University and Hybrid Silica Technologies Inc. have developed a silica nanoparticlebased imaging agent that detects micrometastases in the lymph nodes of two animal models of melanoma. ${ }^{1}$ The nanoparticles can be used for both PET and fluorescence imaging, and the researchers believe it could help noninvasively detect lymph node metastases prior to surgery and, along with handheld devices, guide tumor resection during surgery.

In January, the investigators started a Phase 0 microdosing trial of the nanoparticles in metastatic melanoma patients. The researchers are also forming a new company, Clinical Silica Technologies Inc., to pursue diagnostic and therapeutic applications of the nanoparticle platform.

Michelle Bradbury, who led the team that developed the technology, is the principal investigator for the trial, which will use PET scans to look at pharmacokinetics, safety and dosimetry following systemic injection of the radiolabeled nanoparticles. She is an assistant attending of radiology at Memorial Sloan-Kettering and assistant attending radiologist at Weill Cornell Medical College.

"It will be very exciting to see their Phase 0 results," said Peter Choyke, chief of the Molecular Imaging Program at the National Cancer Institute. "There have been a lot of nanoparticles developed for imaging but few have gone beyond the animal testing stage. The field will be watching how the FDA treats this new type of agent."

Currently, radioactive small molecules are used to detect metastases by PET imaging both prior to surgery and intraoperatively.

The challenge during surgery, said Bradbury, is that "the surgeon can't directly see the radioactive dye, which may limit the extent of disease detection, particularly in areas of complex anatomy such as the head and neck."

Thus, her team wanted to modify a fluorescent nanoparticle with a radiolabel for PET imaging that would allow surgeons to both directly observe tumors using a handheld fluorescence camera during tumor resection and subsequently confirm the optical signal with PET.

The potential advantage of nanoparticles over small molecule imaging agents is the ability to attach more than one type of molecule to the nanoparticle, said Bradbury. However, the difficulty has been developing a nanoparticle with a good blend of tumor targeting, biodistribution and clearance properties.

Bradbury's group began with fluorescent spheres made of silica that were initially developed at Cornell and are now produced by Hybrid Silica Technologies.

The nanoparticles were coated with peptide ligands of integrin $\alpha_{5} \beta_{3}(\mathrm{CD} 49 \mathrm{e} / \mathrm{CD} 61)$ for tumor targeting and radioactive ${ }^{124} \mathrm{I}$ as a PET tracer.

In mice, the particle was cleared through the kidney, with a blood half-time of 5.6 hours. The compound was nontoxic at levels 100 -fold above the proposed human doses.

The researchers next looked to see if the radiolabel on the nanoparticles could be used for noninvasive staging. In a miniswine model of melanoma, injection of radiolabeled nanoparticles followed by whole-body PET imaging identified more metastatic sites in the lymph nodes than a standard whole-body PET scan alone.

Finally, the team studied whether the fluorescent properties of the probe could be used to visualize lymph nodes during surgery. In a xenograft mouse model of melanoma in which the particles were injected near the tumor site prior to surgery, nodes and lymphatic drainage channels could be seen in real time using a fluorescence microscope.

Data were published in The Journal of Clinical Investigation.

\title{
Getting clearance
}

The first hurdle for the technology will be demonstrating that safety and distribution in humans is similar to what was observed in animal models. Of particular importance will be reproducing the kidney clearance data, which is crucial for decreasing background signal and avoiding toxicity.

"There have been a lot of nanoparticles developed for imaging but few have gone beyond the animal testing stage. The field will be watching how the FDA treats this new type of agent."

National Cancer Institute
"The unique thing about this platform is that it targets and clears very quickly," said Bradbury. "The particle is unusual in clearing through the kidney, which helps to limit nonspecific uptake by the liver and other major organs, as well as to reduce organ toxicity and whole-body exposure."

The work is "definitely a step in the right direction, namely, the development of renally cleared, biocompatible nanoparticles," said John Frangioni, professor in the Department of Medicine and Department of Radiology at Harvard Medical School and attending physician in hematology/oncology at Beth Israel Deaconess Medical Center.

However, Frangioni believes the nanoparticles may not be small enough. "The $7 \mathrm{~nm}$ particles described in this paper are slightly too large relative to the renal filtration threshold, resulting in slow elimination from the body," he noted.

Some of the nanoparticles also appear to be sequestered in the spleen, noted Choyke. "It will be important to do good dosimetry in humans and see where this nanoparticle accumulates and how much gets to the target," he said. 


\section{A sharper image}

The major hurdle will be demonstrating that the technology improves on standard of care for either staging melanoma or resecting tumors.

The tumor-specific signal-to-background ratio achieved in the paper is low, said Frangioni. "Although these and other nanoparticles can generate more signal because you're essentially 'filling a bag' with contrast, it comes at the expense of higher background. If the signal washes out before the background clears, one is left with the same (or worse) signal to background that could be achieved using small molecule targeting ligands," he added.

"I am personally not convinced that multimodal imaging, PET and optical in this case, is that important," said Choyke. "First, it makes FDA approval more difficult because there are more components to worry about. More importantly, optical imaging is probably more useful for surgical and endoscopic procedures, whereas the PET is more important in the planning stages. It seems unlikely that those two situations would happen at the same time. So the idea of getting 'two for one', while appealing, may not be that useful."

Bradbury disagreed, noting that "it is important to confirm that the optical signal detected is real and not simply autofluorescence or background signal, particularly in areas of complex anatomy and during the early phases of an investigation. The quantitative nature of PET imaging will provide useful validation for the fluorescence imaging."

"Clinicians are used to PET methods," agreed Tito Gonzalez, VP and head of R\&D at Avelas Biosciences Inc., which develops fluorescence approaches for intraoperative detection of cancerous tumors and metastases. "By having the PET data, you can calibrate the fluorescence data," which may be helpful both preclinically and clinically.

Bradbury told SciBX her team hopes to submit another IND to the FDA within the next year and a half. The new Phase 0 trial would involve injecting metastatic melanoma patients with the nanoparticle locally at a tumor site in the head or neck prior to surgery, followed by imaging metastatic nodes and lymph node drainage patterns with a handheld fluorescence camera system during surgery.
The researchers are working on adapting the nanoparticle for combined use in diagnostic and therapeutic applications. Since ${ }^{124} I$ is already attached, the particle should be readily adaptable as a radiotherapeutic, said Bradbury.

The team also is exploring the possibility of adding a small molecule therapeutic to the nanoparticle to create a targeted therapeutic agent.

A patent application has been filed jointly by Memorial SloanKettering, Cornell and Hybrid Silica Technologies covering composition of matter and methods of use. Clinical Silica Technologies is being founded by investigators from all three organizations, and they "are currently negotiating to license the IP," said Bradbury. Hybrid Silica Technologies will not have a stake in the new company. The licensing terms with Memorial Sloan-Kettering and Cornell are still being negotiated, she added.

Bradbury told SciBX that for the current Phase 0 trial, Hybrid Silica Technologies is producing the core nanoparticle and the radiolabel is being added at Memorial Sloan-Kettering. Manufacturing plans for subsequent clinical trials have not yet been determined.

Kotz, J. SciBX 4(27); doi:10.1038/scibx.2011.757

Published online July 14, 2011

\section{REFERENCES}

1. Benezra, M. et al. J. Clin. Invest.; published online June 13, 2011; doi:10.1172/JCl45600

Contact: Michelle S. Bradbury, Memorial Sloan-Kettering Cancer Center, New York, N.Y.

e-mail: bradburm@mskcc.org

COMPANIES AND INSTITUTIONS MENTIONED

Avelas Biosciences Inc., San Diego, Calif.

Beth Israel Deaconess Medical Center, Boston, Mass.

Clinical Silica Technologies Inc., no location yet

Cornell University, Ithaca, N.Y.

Food and Drug Administration, Silver Spring, Md.

Harvard Medical School, Boston, Mass.

Hybrid Silica Technologies Inc., Ithaca, N.Y.

Memorial Sloan-Kettering Cancer Center, New York, N.Y.

National Cancer Institute, Bethesda, Md.

Weill Cornell Medical College, New York, N.Y. 